\title{
A utilização da fitoterapia e de plantas medicinais em unidades básicas de saúde nos municípios de Cascavel e Foz do Iguaçu - Paraná: a visão dos profissionais de saúde
}

\author{
The use of phytotherapy and medicinal plants \\ in primary healthcare units in the cities of Cascavel \\ and Foz do Iguaçu - Paraná: the viewpoint of health professionals
}

\author{
Maria Cecilia Ribeiro Bruning ${ }^{1}$ \\ Gabriela Bittencourt Gonzalez Mosegui ${ }^{2}$ \\ Cid Manso de Melo Vianna ${ }^{3}$
}

${ }^{1}$ Universidade Paranaense (UNIPAR). R. Rui Barbosa, Centro. 84172-440 Cascavel PR. marycribeiro@unipar.br ${ }^{2}$ Departamento de Saúde e Sociedade, Instituto de Saúde da Comunidade, Universidade Federal Fluminense (UFF).

${ }^{3}$ Instituto de Medicina

Social (IMS), Universidade do Estado do Rio de Janeiro (UERJ).

\begin{abstract}
Phytotherapy and the use of medicinal plants are part of the practice of folk medicine, which complements treatment traditionally employed for the lower income population. This work analyzed the knowledge of health managers and professionals who work in primary health care (PHC) about phytotherapy in the cities of Cascavel and Foz do Iguaçu in the State of Paraná. An exploratory, descriptive, and qualitative study using a structured questionnaire was conducted between February and July 2009. A health manager manifested interest in introducing the program. The other professionals interviewed did not receive training during undergraduate studies or within the Basic Health Units (BHU) where they work. Six professionals (60\%) reported having access to information on phytoterapy through folk wisdom, one (10\%) via training in a BHU, two (20\%) from journals, four (40\%) from the media and four mentioned more than one source. In Foz do Iguaçu, in the BHU where the therapy is deployed, the professionals were not consulted before its implementation. To include phytotherapy in the BHUs of these two cities, it is necessary to train the professionals on the topic, including cultivation and prescription, thereby enhancing the rational use of these medicines.
\end{abstract}

Key words Medicinal plants, Phytotherapy, Health professionals
Resumo A fitoterapia e o uso de plantas medicinais fazem parte da prática da medicina popular, que complementa o tratamento usualmente empregado para a população de menor renda. O trabalho analisou o conhecimento de gestores e profissionais de saúde que atuam na atenção primária (APS), sobre fitoterapia, nos municipios de Cascavel e Foz do Iguaçu, Paraná. Entre fevereiro e julho de 2009, realizou-se estudo exploratório, descritivo, qualitativo, empregando entrevistas $e$ questionário estruturado. Um gestor relatou interesse na implantação do programa, os demais profissionais entrevistados não receberam formação sobre o tema durante a graduação ou nas Unidades Básicas de Saúde (UBS) onde trabalham. Seis profissionais (60\%) relataram ter acesso às informações sobre fitoterapia através do conhecimento popular, uma (10\%) formação na UBS, dois (20\%) através de periódicos, quatro (40\%) através de meio de comunicação e quatro citaram mais que uma das opções. Em Foz do Igua$c ̧ u$, nas UBS onde a terapêutica foi introduzida, os profissionais não foram consultados antes de sua implantação. Para instituir a fitoterapia nas UBS desses dois municípios é necessário capacitar os profissionais quanto ao tema, desde o cultivo até a prescrição, melhorando o uso racional desses medicamentos.

Palavras-chave Plantas medicinais, Fitoterapia, Profissionais de saúde 


\section{Introdução ao tema}

A fitoterapia e o uso de plantas medicinais fazem parte da prática da medicina popular, constituindo um conjunto de saberes internalizados nos diversos usuários e praticantes, especialmente pela tradição oral. Esta prática diminuiu frente ao processo de industrialização, ocorrido no país, nas décadas de 1940 e $1950^{1}$. Trata-se de uma forma eficaz de atendimento primário a saúde, podendo complementar ao tratamento usualmente empregado, para a população de menor renda ${ }^{2}$.

A realização segura desses atendimentos está vinculada ao conhecimento prévio do profissional de saúde sobre a terapêutica com fitoterápicos ou plantas medicinais. A orientação para uma utilização adequada, sem perda da efetividade dos princípios ativos localizados nas plantas e sem riscos de intoxicações por uso inadequado é fundamental ${ }^{3}$.

Schenkel ${ }^{4}$ verificou que além da utilização dos medicamentos alopáticos, a população que busca atendimento nas unidades básicas de saúde (UBS) também utiliza plantas medicinais com fins terapêuticos, muitas vezes desconhecendo a possível existência de efeitos tóxicos, além de não ter entendimento quanto à sua ação terapêutica; qual forma mais correta de cultivo; preparo; quando cada planta pode ser indicada e em quais casos são contraindicadas. Tanto Schenkel ${ }^{4}$ quanto Marques ${ }^{5}$ sugerem a existência de uma crença de não haver nenhum efeito prejudicial à saúde com o emprego de fitoterápicos.

Discussões em torno da implantação da fitoterapia na rede municipal de saúde, ou na atenção primária de saúde, se tornam contraditórias em relação aos preceitos da biomedicina, já que os discípulos desta doutrina se esforçam para eliminar as concepções curativas não baseadas em normas científicas ${ }^{6}$. O crescimento do trabalho desenvolvido com plantas medicinais e fitoterápicos se apresenta como uma alternativa à referência biomédica de saúde, porém, ainda praticamente inexistente nos serviços de saúde tanto públicos como privados ${ }^{7}$.

Observa-se um crescimento na utilização de fitoterápicos pela população brasileira. Dois fatores poderiam explicar este aumento. O primeiro seriam os avanços ocorridos na área científica, que permitiram o desenvolvimento de fitoterápicos reconhecidamente seguros e eficazes. $\mathrm{O}$ segundo é a crescente tendência de busca, pela população, por terapias menos agressivas destinadas ao atendimento primário à saúde ${ }^{8}$.

Porém como apontado por Leite ${ }^{9}$, o interesse por parte dos gestores municipais na implanta- ção de programas de uso de fitoterápicos na atenção primária a saúde, aparece associado apenas à concepção de que esta seria uma opção para suprir a falta de medicamentos. Segundo o autor, só são contabilizados ganhos em custos pela utilização de fitoterápicos e não os benefícios.

A equipe de saúde assiste o paciente e muitas vezes seus familiares nas $\mathrm{UBS}^{6}$. Portanto, com um planejamento adequado de assistência, levando em conta fatores culturais e utilizando os recursos fitoterápicos existentes, pode-se melhorar o nível de saúde da população. Para isso, se faz necessário um conhecimento por parte dos profissionais de saúde que atuam diretamente com os pacientes nas UBS, em relação às propriedades terapêuticas das plantas que são usadas por essa população. Conhecimentos técnicos, que vão desde o preparo para fins terapêuticos, indicações, cuidados e dosagem, e conhecimentos sobre a percepção quanto à relação saúdedoença são imprescindíveis.

\section{Políticas de saúde no Brasil e sua influência nas práticas de saúde}

A Saúde Pública, a cada época, possuiu ligação com movimentos sociais e políticos, além de estar ligada à economia; acabando por seguir as tendências vigentes na sociedade e organizar-se de acordo com as necessidades e com contextos específicos ${ }^{10}$. Para Nunes ${ }^{11}$, a saúde pública se estrutura de acordo com a política nacional de saúde desde o século XX, onde ocorreu a sistematização das práticas sanitárias. A indústria farmacêutica (IF), além das de equipamentos médico-hospitalares também influenciaram o modelo de saúde a ser adotado $^{12}$. No caso do complexo da saúde, é necessário entender que a dinâmica e a evolução dos sistemas de saúde dependem de duas racionalidades, uma interna e outra externa ao sistema. A primeira é definida pela existência de um padrão de acumulação de capital, a partir de duas lógicas distintas - uma pública e outra privada. Já a segunda compreende o conjunto das demandas sobre o sistema de saúde que influenciam o modus operandi do sistema e de seus agentes ${ }^{13}$.

A implantação de um ensino que incorpora os avanços tecnológicos trouxe grandes benefícios no campo da assistência médica. Porém, como o enfoque do modelo hegemônico é na doença, acaba por levar a uma interpretação inadequada do processo saúde- doença, passando este somente ao campo biológico, sendo desconsiderados fatores sociais, culturais ou as causas multifatoriais prováveis. 
“[...] em termos gerais, a produção do conhecimento, neste período, esteve subordinada ao capital e à sociedade do lucro. Neste caso, prevaleceu uma imposição quanto à formação dos profissionais de saúde, que deveria estar em consonância como o mercado de trabalho: na verdade, e da competência desse mercado traçar o perfil desses profissionais, não raro evidenciado incoerências acerca do seu saber-fazer e dos resultados do seu trabalho. O que se tem observado é a mercantilização da saúde, que passa a ter um valor e lucro no mercado capitalista. Dessa forma, a sociedade foi incorporando as formas de assistência à saúde, predominantemente hospitalocêntrica, individual e com alta tecnologia ${ }^{14}$ ".

Os profissionais de saúde acabavam por ter uma formação curativista, proveniente do modelo biomédico, onde valoriza-se dentro da ciência, o desenvolvimento tecnológico, buscando satisfazer uma sociedade altamente consumis$\mathrm{ta}^{14}$. Se o saber científico acaba sendo empregado em detrimento ao saber popular, as práticas de saúde que não são ditas "cientificas", são excluídas. Os cuidados alternativos em saúde passam a não ter valor e surge uma dependência entre os médicos e os medicamentos industrializados, caracterizando o que chamamos de medicalização da sociedade ${ }^{15}$.

A formação dos profissionais contribui para a fragmentação. Aguiar ${ }^{16}$ afirma que nas escolas brasileiras, a exemplo do que acontece em outros países do mundo, existe grande número de estudantes considerados anônimos ou assoberbados de informações fragmentadas, vindas de aulas expositivas de qualidade variável, em processo que valoriza a memorização e não raciocínio crítico.

Os profissionais de saúde não delegam valor ao tratamento por fitoterápicos. Em sua formação acadêmica não receberam conhecimento em relação a essa terapia e acabam criando uma posição de que tudo que é "natureba" não tem efeito farmacológico ${ }^{17}$. Mas acredita-se na cura pelo consumo de medicamentos. O medicamento vira símbolo de saúde, a enfermidade, considerada fator orgânico, é enfrentada mediante seu uso, ficando de lado as esferas social e comportamental ${ }^{18}$.

Em 2004, com intuito de tentar resolver esse quadro de formação superior em saúde, lançouse o "aprender SUS". Esta proposta dos Ministérios da Saúde e da Educação tem como objetivo ampliar a qualidade de vida da população, para que as graduações em saúde passem a valorizar a integralidade, mudando com isso a formação profissional já no âmbito da graduação ${ }^{19}$.
Merhy et al. ${ }^{20}$ propõem, para a democratização da saúde, um modelo que chamam de técnico-assistencial em defesa da vida. A gestão democrática (cogestão entre usuários organizados, prestadores de serviços e governo); saúde como direito de cidadania e serviço publico de saúde voltado para a defesa da vida individual e coletiva, seriam princípios norteadores deste modelo.

Paim $^{21}$ e Merhy et al. ${ }^{20}$, sugerem como estratégias de enfrentamento desta situação, intervenções nos sistemas de saúde, objetivando a implantação de um modelo de assistência que vá além do conceito de saúde como mera cura de doenças, garantindo o direito de cidadania nos serviços públicos que estariam voltados para a defesa da vida. Para esses autores, essa é uma prática complexa a construir-se, que implicará em inúmeros fatores que hoje permeiam as discussões em relação ao SUS.

\section{Sobre a história da utilização dos fitoterápicos}

Nos séculos de colonização, a utilização de plantas medicinais para tratamento das patologias era patrimônio somente dos índios e de seus pajés ${ }^{2}$. A população em geral utilizava medicamentos provenientes de importações, especialmente da Europa. Não existia, ademais, um conhecimento em relação ao correto armazenamento das plantas, a fim de preservar suas propriedades medicinais, ou seja, seus princípios ativos ${ }^{22}$.

Muito tempo foi necessário para que as plantas medicinais do território brasileiro, usadas pelos estrangeiros para tratamento das mais diversas patologias, fossem conhecidas mundialmente. Muitos extratos já eram utilizados em território nacional, desde os primeiros séculos de colonização, para o tratamento de nosologias locais, e, em sua maioria, os medicamentos, utilizados eram fitoterápicos². Para Grams ${ }^{23}$ "[...] a crença popular de que uma simples planta funcionava para tratar doenças aos poucos foi sendo substituída pelo forte apelo dos remédios [...], que causavam certa atração nos pacientes devido à promessa de uma cura rápida e total". Este conceito vem sofrendo modificações já que os fitoterápicos têm conseguido espaço cada vez maior na comercialização de medicamentos.

A ideia de que a utilização de uma gama de formulações para uma única doença deixou de ser viável e a existência de um único medicamento para o tratamento de cada patologia, levou ao surgimento de medicação alopática nas décadas de 1930 e 1940 . Houve a descoberta de que exis- 
tem princípios ativos dentro de cada planta e que a separação desses princípios ativos em forma de medicamento, possibilitava o tratamento das patologias e a cura dos sinais e sintomas característicos de cada uma ${ }^{24}$. Apesar da grande diversidade presente na flora medicinal, o que ocorreu, a partir das décadas citadas anteriormente, foi uma diminuição de incentivos e iniciativas para a continuidade do cultivo e a utilização de plantas como tratamento. Descartava-se uma ampla possibilidade de expansão do uso destas, apesar da diversidade da flora em todo o território nacional. Por falta de desenvolvimento de pesquisas, a maioria dos medicamentos fitoterápicos usados no Brasil passou a ser elaborado a partir de plantas advindas de importação ${ }^{25}$.

Atualmente, percebe-se um desconhecimento entre os profissionais de saúde quanto a esta possibilidade terapêutica. De acordo com Tomazzoni et $a l .{ }^{26}$, há descaso em relação à grande diversidade de tratamentos ainda não desenvolvidos, a partir das plantas existentes no território nacional e particularmente em relação à implantação desta terapêutica no SUS ${ }^{26}$. Para Turolla e Nascimento ${ }^{27}$, ainda que exista um aumento da importância dos fitoterápicos, não existem estudos suficientes para a comprovação da eficácia e segurança da utilização de plantas medicinais como forma de medicamento, que continuam a ser utilizadas com base no conhecimento popular.

Mas foram grandes os progressos nesta área. Yunes et al. ${ }^{8}$ e Ferro ${ }^{28}$ apontam para um cenário promissor, com avanços na área científica que levaram ao desenvolvimento de fitoterápicos seguros e eficazes. Nos países em desenvolvimento, entre eles o Brasil, bem como nos países desenvolvidos, a partir da segunda metade das décadas de 70 e 80 , tem ocorrido um crescimento das "medicinas alternativas", entre elas a fitoterapia ${ }^{29}$.

Matsuda $^{30}$ indica que $50 \%$ dos europeus e mais de $50 \%$ dos norte-americanos fazem uso de fitoterápicos. No Brasil estima-se que o comércio de fitoterápicos seja na ordem de $5 \%$ do total de medicamentos, o que corresponde a mais de US\$ 400 milhões/ano. Gullo e Pereira ${ }^{31}$ com base em dados da OMS apontam que, em 1980, o mercado mundial de fitoterápicos e produtos naturais movimentou 500 milhões de dólares. Para o ano de 2000, a previsão era de que somente na Europa registrar-se-ia um volume de vendas de $500 \mathrm{mi}$ lhões de dólares. No Canadá as vendas crescem $15 \%$ ao ano e nos Estados Unidos chegam a 20\%. Esse aumento de consumo de plantas medicinais deve-se a vários fatores. Para Lorenzi e Matos ${ }^{32}$, as preocupações com a biodiversidade e as ideias de desenvolvimento sustentável despertaram interesse no uso das plantas. Neste trabalho, e também no realizado por Matsuda ${ }^{30}$, relata-se que a população busca uma melhor qualidade de vida, com métodos mais naturais e saudáveis para a manutenção da saúde. Já para Simões et al..$^{33}$, o interesse na utilização de plantas medicinais decorre também do o alto custo dos medicamentos industrializados, da crise econômica e da falta de acesso à assistência médica e farmacêutica, fatores que geram a procura dos consumidores por utilizar produtos naturais.

A população passa a questionar o uso indiscriminado de medicamentos sintéticos e procura, alternativamente, os fitoterápicos. Além de todos esses fatores, a ação terapêutica tem sido comprovada com muitas plantas utilizadas popularmente. Leite 9 assim resume a discussão: a “[...] insatisfação com o sistema de saúde oficial, a necessidade das pessoas da sensação de controle de seu próprio corpo e recuperação da saúde, tomando as práticas de saúde por si ou para sua família [...]", são fatores que estão contribuindo para o aumento da utilização de fitoterápicos ${ }^{9}$.

Segundo Jorquera ${ }^{34}$ e Alves e Silva ${ }^{35}$, a Organização Mundial da Saúde (OMS) estima que 80\% da população mundial não possuí acesso ao atendimento primário em saúde. Na America Latina, $50 \%$ da população têm pouco ou nenhum acesso aos medicamentos, mas ainda é marginal o diálogo da política de saúde e o uso de fitoterápicos na atenção básica no SUS ${ }^{36}$.

\section{Fitoterapia na atenção primária à saúde}

Na Conferência Internacional sobre Cuidados Primários de Saúde, realizada em 1978, como parte do plano de ação da OMS para atender o objetivo "Saúde para todos no ano 2000", foi incentivada a valorização das terapias tradicionais, entre elas a fitoterapia, reconhecidas como recursos possíveis, mais fáceis e economicamente viáveis de aumentar a cobertura de atenção primária à saúde ${ }^{37}$.

No Brasil, este tema foi discutido na $8^{a}$. Conferencia Nacional de Saúde, em 1986, quando se recomendava a introdução de práticas tradicionais de cura popular no atendimento publico de saúde ${ }^{38}$. Durante a 10 a Conferência Nacional de Saúde, em 1996, houve a proposta de incorporar no SUS as terapias alternativas e práticas populares, especificamente o incentivo à fitoterapia e à homeopatia na assistência farmacêutica públi$\mathrm{ca}^{2}$. O uso da fitoterapia na atenção primária é tecnicamente apropriado, quando a prescrição 
não é feita de forma indiscriminada e quando os profissionais não incentivam a medicalização excessiva $^{39}$. A atenção às possibilidades alternativas de tratamento levaria a uma melhora no atendimento da população pelo Sistema Único de Saúde, em razão de proporcionar outra forma de tratamento e de prevenção.

Leite $^{9}$ resume, novamente, os pontos mais salientes dessa discussão:

“[...] o interesse por parte de gestores municipais na implantação de programas de uso de fitoterápicos na atenção primária a saúde, muitas vezes aparece associado apenas à concepção de que esta é uma opção para suprir a falta de medicamentos na impossibilidade de disponibilização destes, já que na maioria das vezes contabilizam-se os ganhos em custos gerados pela utilização dos fitoterápicos" 9 .

Este autor afirma que além da viabilidade econômica e da ação terapêutica, a fitoterapia representa parte importante da cultura de um povo, que não pode ser desconsiderada. No entanto, "a utilização de plantas medicinais tem sido muitas vezes considerada como fato desvinculado da assistência à saúde como um todo e vista como simples medicamentação" .

$\mathrm{Na}$ mesma direção, Hufford ${ }^{40}$ pontua que "um sistema de saúde que adota a fitoterapia deve incorporar um conjunto de atitudes, valores e crenças que constituem uma filosofia de vida e não meramente uma porção de remédios”. Akerele ${ }^{41}$ sinaliza que o saber popular pode ser uma contribuição aos profissionais de saúde, na implantação de programas de fitoterapia na rede de atendimento básico de saúde. Torna-se possível, também, desenvolver o potencial econômico em relação às plantas com valor medicinal. A rede básica de atendimento a saúde deve proporcionar aos pacientes todos os recursos apropriados e disponíveis de assistência. O uso adequado das plantas medicinais é uma alternativa de tratamento à medicina alopática, mas não deve ser considerado um substituto desta ${ }^{41}$.

Para que o conhecimento científico seja mais bem difundido e se torne uma realidade, necessita-se de projetos tecnológicos e pesquisas que possam fundamentar e fortalecer a fitoterapia e possam fazer com que a Política Nacional de Plantas Medicinais e Medicamentos Fitoterápicos seja implementada em todo o território nacional, especialmente nas $\mathrm{UBS}^{17}$. Está em jogo o que preceitua a política de desenvolvimento tecnológico, para a saúde:

"Deverá ser continuado e expandido o apoio à pesquisa que vise o aproveitamento do poten- cial terapêutico da flora e fauna nacionais, enfatizando a certificação de suas propriedades medicamentosas" 42 .

Espera-se que a implantação dessa política de medicamentos fitoterápicos nos locais de atenção primária que atendem o SUS permita uma redução nos gastos com saúde no Brasil. O grande problema enfrentado pelas UBS, que sofrem com a falta de medicamento, poderá ser amenizado quando efetuada a complementação do medicamento convencional pelo fitoterápico, mediante uma orientação adequada ${ }^{17}$.

No Paraná, uma experiência com programa de fitoterápicos na rede pública deu-se no município de Curitiba, onde as plantas medicinais e os fitoterápicos são usados como recurso terapêutico desde 1990. Neste município existe o projeto Verde Saúde, que preconiza a utilização de plantas medicinais. Em 2001, 82\% das unidades de saúde já utilizavam a fitoterapia como opção terapêutica ${ }^{43}$. Outros municípios paranaenses implantaram o programa de uso de fitoterápicos na rede básica. Londrina, Toledo e Foz do Iguaçu avaliam os resultados desse programa como satisfatórios para o serviço e para os usuários do Sistema Público de Saúde ${ }^{17}$.

A equipe de saúde assiste ao paciente, à família e à comunidade, planeja a assistência com base na cultura da população, utiliza os recursos disponíveis, ajudando dessa forma a comunidade a melhorar seu nível de saúde. Para isso, necessita de conhecimento das propriedades terapêuticas das plantas a serem usadas, do preparo, da indicação, dos cuidados e da dosagem ${ }^{6}$.

De acordo com Furnham e Bragrath ${ }^{44}$, o uso de plantas com fins medicinais vem de uma população insatisfeita, muitas vezes devido a reações adversas causadas pelos medicamentos; ou ainda da busca por soluções alternativas para as doenças. Com isso, a prática do uso de plantas permanece presente, dentro do crescente uso das terapias não convencionais. Mas, ainda existe uma crença difundida de que o que é natural não faz mal, que as plantas apenas curam, o que acaba por direcionar ainda mais ao consumo e à aceitação das terapias alternativas, especialmente da fitoterapia o que ainda é referido pelos mesmos autores.

A fitoterapia apresenta-se como uma possibilidade de atuar como coadjuvante nos tratamentos alopáticos, desde que sejam levadas em consideração suas possíveis complicações. Fazse necessário um conhecimento por parte dos profissionais de saúde que estarão atuando e indicando a terapia aos pacientes. Essas considera- 
ções, entre muitas outras, levaram ao desenvolvimento deste trabalho, que buscou levantar desde aspectos da implantação da política de fitoterápicos até a extensão dos conhecimentos dos profissionais de saúde, em relação à fitoterapia nas UBS. A experiência já está presente nos municípios de: Londrina, Maringá, Curitiba, Toledo e Foz do Iguaçu e ainda não foi implantada no município de Cascavel, no estado do Paraná.

Esta pesquisa teve por intenção servir de apoio e incentivo à implantação da fitoterapia nas UBS como atendimento primário, visando à melhora da qualidade de vida aos usuários do SUS. Esta terapêutica, quando baseada em sólidos conhecimentos científicos, amplia o acesso às populações carentes, afetadas por não terem condições de adquirir os medicamentos industrializados, não disponíveis no SUS. Este trabalho pretendeu ser objeto de inspiração para novas pesquisas na área de atuação da saúde, a fitoterapia, visando uma possível ampliação do uso de fitoterápicos na rede básica de saúde, a fim de aumentar o acesso a medicamentos na atenção primária.

\section{Materiais e metodologia}

Este trabalho foi dividido em duas etapas. $\mathrm{Na}$ primeira foi realizada uma revisão científica da literatura. O método de levantamento bibliográfico baseou-se na busca de artigos de periódicos, teses, dissertações e capítulos de livros, nacionais e internacionais, indexados em bases eletrônicas, tais como: Scielo, Lilacs, Medlinee Portal Capes, no período de 28 anos (1980 a 2008). As seguintes palavras-chave foram empregadas como base de pesquisa: fitoterapia (phytoteraphy, fitoterápia), atenção primária à saúde (primary health care, atencion primária a la salud).

Dentre os 1560 estudos encontrados, somente se considerou os artigos nos quais fosse possível a identificação com a utilização das plantas medicinais e fitoterápicos na saúde básica e a ligação com o conhecimento dos profissionais de saúde, restando portanto, um numero de 150 artigos. Adicionalmente, foram utilizados para a pesquisa as referencias citadas nos artigos selecionados, no intuito de captar artigos que não foram identificados na busca eletrônica.

A segunda etapa constituiu-se por pesquisa de campo realizada em dois municípios da região oeste do Paraná. O objetivo desta etapa era averiguar, entre os dez profissionais de saúde nos dois municípios dentre eles, cinco enfermeiros, três médicos, uma auxiliar de enfermagem e uma técnica em enfermagem a formação profissional, o nível de especialização, qual tipo de vinculo com a Unidade Básica de Saúde, regime de trabalho, tempo de trabalho na UBS, se houve formação na graduação ou pós-graduação sobre plantas medicinais e fitoterápicos, como foi o acesso aos conhecimentos sobre fitoterapia, quantos profissionais de saúde estão envolvidos no atendimento à população, entre outros itens. Esta etapa realizou-se através de um roteiro de entrevista de natureza estruturada, ou seja, um roteiro com perguntas elaboradas previamente pela pesquisadora antes de ir a campo, o que permitiu flexibilidade e o aprofundamento de assuntos que poderiam vir a surgir ${ }^{45}$.

Escolheram-se Unidades Básicas de Saúde que já possuem a terapia implantada e outras que ainda não possuem nos municípios de Cascavel e Foz do Iguaçu. A ideia de enfoque nas UBS fundamentou-se no fato de que as patologias que não são caracterizadas como emergência ou urgências levam a população a buscar o atendimento primário nestes locais e plantas medicinais e fitoterápicos poderiam ser fornecidos por estas unidades, em conjunto com os medicamentos alopáticos.

Após aprovação pelo comitê de ética em pesquisa da Universidade do Estado do Rio de Janeiro (UERJ), procedeu-se à aplicação dos questionários nas próprias UBS, em horários previamente agendados, juntamente com um termo de consentimento que foi preenchido pelo entrevistado. O levantamento foi realizado do período de fevereiro a julho de 2009.

\section{Resultados}

Um total de dez profissionais de saúde nos dois municípios dentre eles, cinco enfermeiros, três médicos, uma auxiliar de enfermagem e uma técnica em enfermagem (Gráfico 1), participaram da pesquisa sendo que os itens pesquisados foram a formação profissional, o nível de especialização, qual tipo de vinculo com a Unidade Básica de Saúde, regime de trabalho, tempo de trabalho na UBS, se houve formação na graduação ou pós-graduação sobre plantas medicinais e fitoterápicos, como foi o acesso aos conhecimentos sobre fitoterapia, quantos profissionais de saúde estão envolvidos no atendimento a população, entre outros itens.

A partir dos dados obtidos nas entrevistas foram construídas algumas tabelas e gráficos com o auxilio do programa Microsoft Excel dando 
ênfase a diversos aspectos como: profissionais envolvidos no atendimento aos pacientes das unidades básicas visitadas, forma como obteve o conhecimento sobre plantas medicinais e fitoterápicas, aceitação ou não do uso de plantas como complemento dos tratamentos dos pacientes. Lançou-se mão de um processo de tradução e decodificação dos dados obtidos ao longo do trabalho de campo (dados êmicos) para a linguagem científica (dados éticos) com a finalidade de tornar a informação mais compreensível ao universo acadêmico e facilitar o agrupamento e a padronização dos dados para comparações futuras. O ponto de vista êmico corresponde ao modelo particular do sistema em estudo, já o ético corresponde a um nível de análise e discussão não necessariamente relacionado ao sistema estudado, e sim a um nível "neutro" de descrição e análise pertencente ao pesquisador ${ }^{46}$.

\section{Discussão}

Esta pesquisa foi realizada com profissionais de saúde que trabalham nas Unidades Básicas de Saúde dos municípios de Cascavel e Foz do Iguaçu. Durante a observação participante, foi possível perceber questões sociais de relevância, para a compreensão do significado dos saberes e práti-

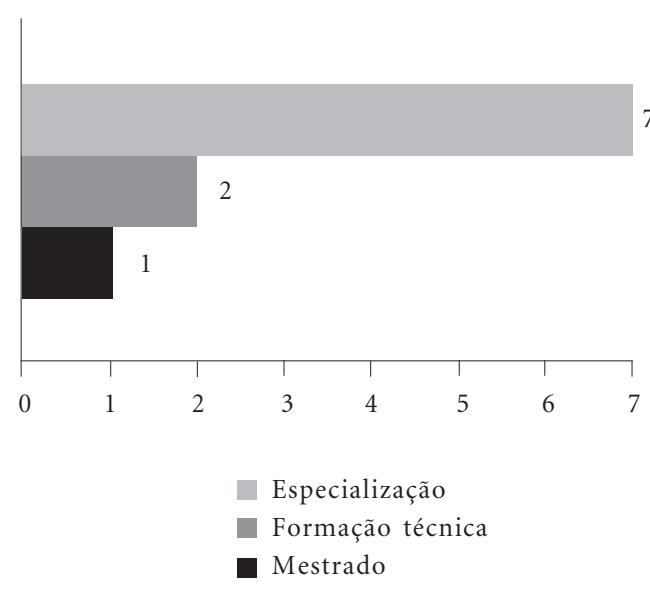

Gráfico 1. Nível de formação profissional dos profissionais de saúde e gestores das Unidades Básicas dos Municípios de Cascavel e Foz do Iguaçu $-\mathrm{PR}$.

Fonte: elaboração própria (2009). cas em plantas medicinais do ponto de vista dos profissionais de saúde das Unidades Básicas.

Foram realizadas 10 entrevistas, sendo $03 \mathrm{em}$ Foz do Iguaçu e 7 em Cascavel, o que representou a totalidades dos participantes dos grupos no período da pesquisa. Do total dos entrevistados $80 \%$ eram mulheres e $20 \%$ eram homens.

Nas entrevistas, procurou-se saber sobre a origem do conhecimento em plantas medicinais e a transmissão do mesmo. Observou-se uma diversidade nas formas de apreensão e transmissão do conhecimento devido às diferenças culturais e socioeconômicas entre os profissionais de saúde. Estas foram: transmissão oral dentro do núcleo familiar, pelo rádio, televisão, livros e até pela internet. Dos entrevistados seis relataram o acesso às informações sobre fitoterapia através do conhecimento popular, um formação na unidade básica de saúde, dois através de periódicos, quatro através de meio de comunicação, sendo que quatro citaram mais que uma das opções (Gráfico 2).

Estudo realizado por Alves e Silva ${ }^{35}$, demonstrou que o conhecimento das plantas medicinais por parte de uma comunidade vinha $11 \%$ através de vizinhos, $8 \%$ com profissionais de saúde, 5\% de acordo com crenças ou tradições, 57\%

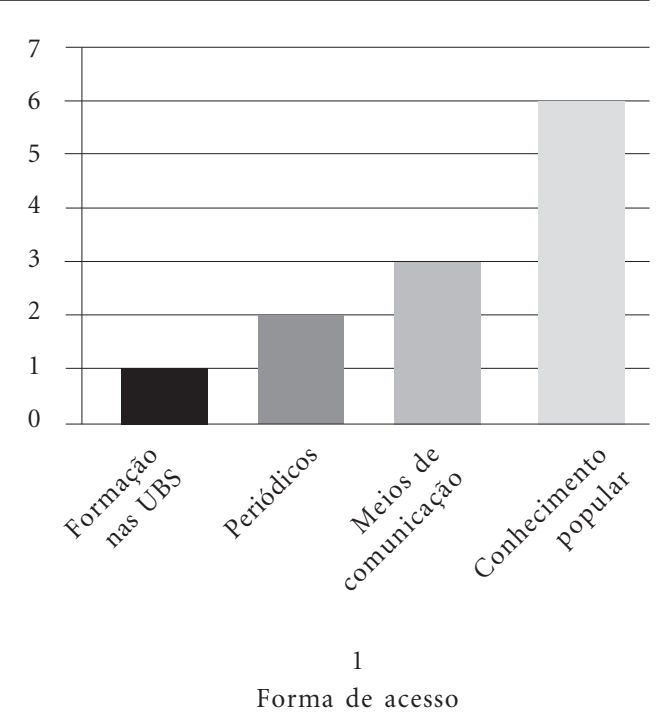

Gráfico 2. Forma de acesso dos profissionais de saúde ao conhecimento sobre a fitoterapia.

Fonte: elaboração própria (2009). 
com pais ou avós, $1 \%$ em livros ou revistas 18\% em outras fontes. Outro trabalho realizado por Barbosa $^{47}$, mostra que graduandos de enfermagem questionados sobre o modo como ficaram conhecendo as terapias alternativas, referiram com maior frequência (33 vezes, o que significa $34,37 \%$ dos graduandos entrevistados) livros, revistas e televisão; 27 graduandos $(28,12 \%)$ referiram amigos e familiares; 21 (21,87\%), pessoas que as utilizavam; 14 ( $14,58 \%)$ afirmaram conhecer pois as utilizavam ou haviam utilizado.

Esta multiplicidade de fontes, formas e origem do aprendizado, ligada a saberes tradicionais ou atuais, se encontram justapostos uns aos outros, se complementando, para a formação de um modelo único, novo e diferente das influências anteriores. Este fenômeno cultural é característico da pós-modernidade globalizada e estão seguramente presentes na Medicina Popular nos grandes centros urbanos atualmente ${ }^{48}$.

Após uma pesquisa a respeito das patologias mais frequentes nas unidades de saúde ${ }^{49}$, a equipe do Programa de Fitoterapia do município de Foz do Iguaçu selecionou algumas plantas medicinais que apresentavam segurança e eficácia comprovadas na literatura. Atualmente, o programa conta com um elenco de 10 plantas organizadas no Memento Terapêutico ${ }^{50}$ e manipula cápsulas além de indicar chás.

Do total dos entrevistados apenas duas (médica e enfermeira) das Unidades Básicas de Foz do Iguaçu fazem a prescrição dos medicamentos aos seus pacientes, nas Unidades básicas de Ouro Verde (médica) e Padre Monti (enfermeira) em Foz do Iguaçu. Os demais profissionais só fazem uso de forma caseira através do conhecimento popular. A média de trabalho nas Unidades Básicas dos profissionais foi de três dias até 20 anos, sendo o regime de trabalho dos profissionais de 20 a 40 horas (Gráficos 3 e 4).

Dos profissionais de saúde entrevistados apenas duas possuíam treinamento para prescrever plantas medicinais a seus pacientes, pois as prefeituras não oferecem esse treinamento aos trabalhadores das UBS, sendo um fato lamentável que estes estejam pouco preparados para lidar com as plantas, pois deveriam ser propostos subsídios para quem atua na saúde comunitária em relação ao uso medicinal das plantas e derivados ${ }^{3}$.

Segundo Nuñez e Ciosak ${ }^{51}$, há necessidade de buscar novos saberes como opções de assistência à promoção da saúde da população, devido à deficiência de aquisição de conhecimento durante a graduação dos profissionais de saúde em geral.

Quando questionados sobre a implantação de uso de fitoterapia e plantas medicinais em todas as Unidades Básicas nos Municípios pesquisados os profissionais foram unânimes a favor desta medida, acreditando que isto auxilia na saúde geral da população por não apresentarem efeitos adversos especialmente quando utilizadas as plantas já conhecidas e fornecidas pela Itaipu

Gráficos 3 e 4. Regime de trabalho dos profissionais de saúde dos municípios de Cascavel e Foz do Iguaçu segundo carga horária.
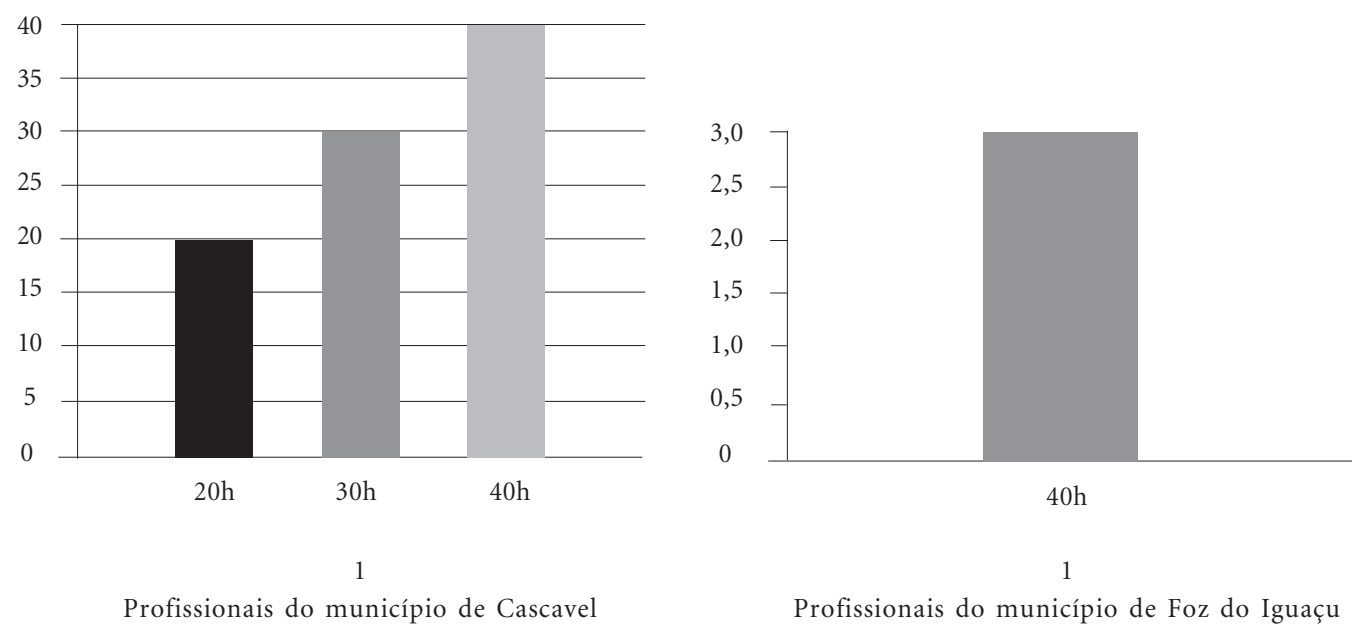

1

Profissionais do município de Foz do Iguaçu 
Binacional. Resultado semelhante ao encontrado por Tomazzoni et al. ${ }^{26}$ ao realizarem uma entrevista nas Unidades Básicas do Município de Cascavel, em que 100\% dos entrevistados responderam favoravelmente sobre a possibilidade de serem prescritas plantas medicinais nas UBS em substituição aos medicamentos industrializados, descrevendo várias justificativas, tais como: acreditar que as plantas têm poder de cura; preferirem utilizar produtos naturais por terem menos efeitos colaterais, entre outras afirmações.

Dutra $^{52}$ realizou pesquisa com 220 profissionais da área de saúde que atuam em unidades básicas de Anápolis, Estado de Goiás, quando questionados sobre a posição em relação ao uso de plantas medicinais e fitoterápicos dentro das UBS, os mesmos apresentaram postura divergente, conforme sua formação acadêmica de base. Enquanto todos os fisioterapeutas, farmacêuticos e odontólogos se posicionaram favoravelmente $(100 \%)$, entre os médicos o percentual foi de apenas $17 \%$. Entre os enfermeiros, $65 \%$ manifestaram-se favoráveis; já os técnicos de enfermagem manifestaram-se amplamente favoráveis (86\%).

No transcorrer do presente estudo, percebeuse que a utilização das plantas na terapia popular, no Município de Foz do Iguaçu, é bastante difundida e presente, sendo que a transferência do conhecimento etnobotânico, neste local, segue as orientações repassadas pela Itaipu Binaci- onal através de seu programa, e que as plantas nas Unidades Básicas deste Município representam papel importante na manutenção das condições de saúde, constituindo também parte de um saber local preservado e utilizado, ou seja, de sua cultura e costumes.

A utilização de plantas medicinais vem ao encontro das proposições da Organização Mundial da Saúde (OMS), que incentiva a valorização das terapias tradicionais, reconhecidas como recursos terapêuticos úteis, podendo atender a algumas demandas de saúde da população, além de contribuir para o sistema local de saúde dos usuários do sistema publico de saúde ${ }^{37}$.

\section{Conclusão}

A diversificação das praticas de atendimento a saúde é de conveniência acadêmica, socioeconômica e cultural, sendo assim, a aplicação das plantas medicinais e fitoterápicas é um possível meio de ampliação da área de trabalho dos profissionais de saúde que ainda estão pouco informados e preparados para lidar com esses recursos alternativos. Para que os profissionais conheçam melhor essas praticas e possam aplicá-las de maneira coerente no serviço publico de saúde se faz importante a inclusão destes conhecimentos nas atividades de ensino, pesquisa e extensão.

\section{Colaboradores}

MCR Bruning e GBG Mosegui trabalharam na concepção, metodologia e aprovação da versão final; e, CMM Vianna na metodologia, revisão e aprovação da versão final. 


\section{Referências}

1. Bragança ALR. Plantas medicinais antidiabéticas: uma abordagem multidisciplinar. Niterói: EDUFF; 1996.

2. Eldin S, Dunford A. Fitoterapia na atenção primária a saúde. São Paulo: Manole; 2001.

3. Arnous AH, Santos AS, Beinner RPC. Plantas medicinais de uso caseiro- conhecimento popular e interesse pelo cultivo comunitário. Espaç. saúde 2005; 6(2):01-06.

4. Schenkel EP. Cuidado com os medicamentos. As plantas medicinais, os chás e os fitoterápicos. Porto Alegre: Saga, Deluzzata; 1995.

5. Marques FC. Fito 2000 - Lima, Peru. Boletim da Associação Catarinense de Plantas Medicinais. No prelo 2001.

6. Araújo M. Das ervas medicinais à fitoterapia. São Paulo: Ateliê Editorial; 2002.

7. Guizardi FL, Pinheiro R. Novas práticas sociais na constituição do direito à saúde: a experiência de um movimento fitoterápico comunitário. Interface Comun saúde Educ 2008; 12(24):109-122.

8. Yunes RA, Pedrosa RC, Cechinel FV. Fármacos e fitoterápicos: a necessidade do desenvolvimento da indústria de fitoterápicos e fitofármacos no Brasil. Quimica Nova 2001; 24(1):147-152.

9. Leite SN. Além da medicação: a contribuição da fitoterapia para a saúde publica [dissertação]. São Paulo (SP): Universidade de São Paulo; 2000.

10. Luz MT. Notas sobre as políticas de saúde no Brasil de transição demográfica - Anos 80. In: Santana JP, Organizador. Desenvolvimento gerencial de unidades básicas de saúde do distrito sanitário. Brasília: Opas; 1995. p. 138-154.

11. Nunes ED. Sobre a história da saúde pública: idéias e autores. Cien Saude Colet 2000; 5(2):251-264.

12. Cordeiro HA. A indústria da saúde no Brasil. Rio de janeiro: Graal; 1980.

13. Vianna CMM. Estruturas do Sistema de Saúde: do Complexo Médico-industrial ao Médico-financeiro. Physis 2002; 12(2):375-390.

14. Oliveira EM, Aragão AEA. A influência positivista na formação dos profissionais de saúde. [artigo da internet]. [acessado 2008 ago 14]. Disponível em: http://sbmfc.org.br/site/bib/influenciapositiva.pdf

15. Siqueira JE. Tecnologia e medicina entre encontros e desencontros. Revista de Bioética 2000; 8(1):55-65.
16. Aguiar ACA. Implementando as novas diretrizes curriculares para a educação médica: o que nos ensina o caso de Harvard? Interface Comun Saude Educ. 2001; 5(8):161-166.

17. Tomazzoni MI. Subsídios para a introdução do uso de fitoterápicos na rede básica de saúde do Município de Cascavel/PR [dissertação]. Curitiba: Universidade Federal do Paraná; 2004.

18. Lefèvre F. A função simbólica dos medicamentos. Rev Saude Publica 1983; 17(6):500-503.

19. Ministério da Saúde (MS). Aprender SUS: o SUS e os cursos de graduação na área da saúde 2004. [documento da internet]. [acessado 2008 ago 25]. Disponível em : http://www.saude.gov.br.

20. Merhy EE, Cecilio LCO, Nogueira FRC. Por um modelo tecno-assistencial da política de saúde em defesa da vida: contribuição para as conferências de saúde. Saúde em Debate 1991; 33:83-89.

21. Paim JSA. Reforma sanitária e os modelos assistenciais. In: Rouquayrol MZ, organizador. Epidemiologia e Saúde. 5a Edição. Rio de Janeiro: MEDSI; 1999. p. 473-487.

22. Martins ER, Castro DM, Castellani DC, Dias JE. Plantas medicinais. Viçosa: Universidade Federal de Viçosa; 2000.

23. Grams WFMP. Plantas medicinais de uso popular em cinco distritos da Ilha de Santa Catarina - Florianópolis, SC [dissertação]. Curitiba: Universidade Federal do Paraná; 1999.

24. Brasil. Ministério da Saúde (MS), Secretaria de Políticas de Saúde. Política nacional de medicamentos. Brasília: MS; 1999.

25. Lima JJF. Perspectivas dos cirurgiões dentistas sobre a inserção da fitoterapia na atenção básica de saúde [dissertação]. Natal: Universidade Federal do Rio Grande do Norte; 2005.

26. Tomazzoni MI, Negrelle RRB, Centa M.L. Fitoterapia popular: a busca instrumental enquanto prática terapêutica. Texto Contexto enfermagem 2006; 15(1):115-121.

27. Turolla MSR, Nascimento ES. Informações toxicológicas de alguns fitoterápicos utilizados no Brasil. Revista Brasileira de Ciências Farmacêuticas 2006; 42(2):289-306. 
28. Ferro D. Fitoterapia: conceitos clínicos. São Paulo: Atheneu; 2008.

29. Luz MT. Cultura contemporânea e medicinas alternativas: novos paradigmas em saúde no fim do século XX. Physis 1997; 7(1):13-43.

30. Matsuda AH . Fitoterápicos: complementos nutricionais ou medicamentos? In: Torres EAFS, organizador. Alimentos do milênio: a importância dos transgênicos, funcionais e fitoterápicos para a saúde. São Paulo: Signus; 2002. p. 31-41.

31. Gullo C, Pereira C. De volta à inquisição. Revista Isto É 1998 set; p. 128-130.

32. Lorenzi H, Matos FJA. Plantas medicinais no Brasil: nativas e exóticas. São Paulo: Nova Odessa; 2002.

33. Simões CMO, Mentz LA, Schenkel EP, Irgang BE, Stehmann JR. Plantas da medicina popular no Rio Grande do Sul. Porto Alegre: UFRGS; 1988.

34. Jorquera CS. Utilización industrial de plantas medicinales. In: Workshop presented in UNIDO in Latin America; 1993 jul 11-17; Guatemala; 1993.

35. Alves DL, Silva CR. Fitohormônios: abordagem natural da terapia hormonal. São Paulo: Atheneu; 2002.

36. Teixeira JBP. Estratégia de proteção patentária para plantas Medicinais e Medicamentos Fitoterápicos em adição aos Genéricos no SUS [tese]. Rio de Janeiro: Universidade do Estado do Rio de Janeiro; 2005.

37. Organização Pan-Americana da Saúde. Declaração de Alma-Ata. In: Conferência Internacional Sobre Cuidados Primários de Saúde, 6-12 set 1978, AlmaAta. [site da Internet]. [acessado 2009 ago 04]. Disponível em: http://www.opas.org.br/promocao/ uploadArq/Alma-Ata.pdf.

38. Brasil. Ministério da Saúde (MS). Ministério da Previdência e Assistência Social. Boletim da 8a. Conferencia Nacional de Saúde. Brasília: MS; 1986.

39. Espirito Santo. Programa de fitoterapia. [artigo na Internet]. [acessado 2008 ago 29]. Disponível em www.vitoria.es.gov.br/secretarias/saude/fito1.htm.

40. Hufford DJ. Folk medicine and health culture in contemporary society. Prim Care 1997; 24 (4):723741.

41. Akerele O. Medicinal plants and primary health care: an agenda for action. (tradução de André A. R. de Meijer e Ceres B. Laus). Revista de Fitoterapia 1988; 59(5):355-363.
42. Brasil. Ministério da Saúde (MS), Departamento de Atenção Básica. Guia prático de saúde da família. Brasilia: MS; 2001.

43. Curitiba. Secretaria Municipal de Saúde. Programa de fitoterapia 2003. [artigo na Internet]. [acessado 2008 ago 29]. Disponível em: http://www. plantas medicinais.org/saúde/arquivo000013.html

44. Furnham A, Bhagrath R. A comparison of health beliefs and behaviours of clients of orthodox and complementary medicine. Br J Clin Psychol 1993; 32(2):237-246.

45. Albuquerque UP. Introdução à Etnobotânica. Recife: Edições Bagaço; 2002.

46. Geertz C. O saber local: novos ensaios em antropologia interpretativa. 5a Edição. Petrópolis: Vozes; 2002.

47. Barbosa, MA. A utilização de terapias alternativas por enfermeiros brasileiros. [tese]. Universidade de São Paulo: São Paulo; 1994.

48. Luz MT. Novos saberes e práticas em saúde coletiva: Estudo sobre racionalidades médicas e atividades corporais. 2a Edição. São Paulo: Hucitec; 2005.

49. Santos MAP, Reis MCP. Relato de uma experiência de incentivo ao uso popular da planta medicinal dentro de uma prática médica generalista numa comunidade adstrita (ilha de Paquetá - Rio de Janeiro). In: XV Simpósio de Plantas Medicinais do Brasil, 1998; Águas de Lindóia, São Paulo; 1998.

50. Rio de Janeiro. Secretaria Municipal de Saúde (SMS). Memento Terapêutico: Programa de Fitoterapia. Rio de Janeiro: SMS; 2002.

51. Nuñez, HM, Ciosak, SI. Terapias alternativas/complementares: o saber e o fazer das enfermeiras do distrito administrativo 71 - Santo Amaro - São Paulo. Rev Enf USP 2003; 37(3):11-18.

52. Dutra MG. Plantas Medicinais, Fitoterápicos e saúde pública: um diagnóstico situacional em Anápolis, Goiás. [dissertação]. Anápolis: UniEvangélica; 2009.

Artigo apresentado em 30/06/2011

Aprovado em 25/08/2011

Versão final apresentada em 13/12/2011 
